\title{
A NOTE ON PERIODIC POINTS AND RECURRENT POINTS OF MAPS OF DENDRITES
}

\author{
Hisao KaTo
}

Let $f: X \rightarrow X$ be a map of a continuum $X$. Let $P(f)$ denote the set of all periodic points of $f$ and $R(f)$ denote the set of all recurrent points of $f$. In [2], Coven and Hedlund proved that if $f: I \rightarrow I$ is a map of the unit interval $I=[0,1]$, then $\mathrm{Cl}(P(f))=\mathrm{Cl}(R(f))$. In [7], Ye generalised this result to maps of a tree. It is natural to ask whether the result generalises to maps of a dendrite. (A dendrite is a locally connected continuum which contains no simple closed curve.) The aim of this paper is to show that the answer is negative.

\section{INTRODUCTION}

All spaces considered in this paper are assumed to be separable metric spaces. By a continuum, we mean a nonempty compact connected metric space. Let $I$ be the unit interval $[0,1]$. A tree is a 1-dimensional connected compact polyhedron which contains no simple closed curve. A continuum $X$ is a dendrite if $X$ is a locally connected continuum and $X$ contains no simple closed curve. (See [5] for topological properties of dendrites.) Note that $X$ is a dendrite if and only if $X$ is a 1-dimensional compact absolute retract. The dynamics of maps (=continuous functions) of $I$ and trees are fairly well understood. The dynamical behavior of maps of dendrites have often appeared as Julia sets in complex dynamical systems (for example, see [6]).

Let $X$ be a compact metric space with metric $d$ and $f: X \rightarrow X$ a map. A point $x \in X$ is a periodic point of $f$ if there is a natural number $n \geqslant 1$ such that $f^{n}(x)=x$. A point $x \in X$ is a recurrent point of $f$ if for each $\varepsilon>0$ there is a natural number $n \geqslant 1$ such that $d\left(f^{n}(x), x\right)<\varepsilon$. By $P(f)$, we mean the set of all periodic points of $f$, and by $R(f)$ the set of all recurrent points of $f$. The notions of periodic points and recurrent points are very important in the study of dynamical systems. Coven and Hedlund [2] proved that if $f: I \rightarrow I$ is any map, then $\operatorname{Cl}(P(f))=\operatorname{Cl}(R(f))$. This result was generalised by Ye to maps of trees [7]. It is natural to ask whether the result can be generalised to maps of dendrites. The aim of this paper is to show that the answer is negative.

Received 11th July, 1894

Copyright Clearance'Centre, Inc. Serial-fee code: 0004-9729/95 \$A2.00+0.00. 


\section{Dendrites with $\mathrm{Cl}(P(f)) \neq \mathrm{Cl}(R(f))$}

In this section, we construct a dendrite $D$ and a map $f: D \rightarrow D$ such that $\mathrm{Cl}(P(f)) \neq \mathrm{Cl}(R(f))$.

First, we construct some kinds of dendrites summarising the general method of Krasinkiewicz [4], as follows. Let $X$ be any compact metric space and let $g: X \rightarrow X$ be any map of $X$. Choose an inverse sequence $X=\left\{X_{n}, p_{n, n+1} \mid n=1,2, \ldots\right\}$ of compact polyhedra $X_{n}$ such that $X_{1}=\{*\}$ is a one point set, $p_{n, n+1}: X_{n+1} \rightarrow X_{n}$ is a bonding map $(n \geqslant 1)$ and $X=\operatorname{invlim} X$. For $1 \leqslant m<n$, let $p_{m, n}=p_{m, m+1} \cdots p_{n-1, n}$ and let $p_{n}: X \rightarrow X_{n}$ be the natural projection. Now, consider the infinite telescope $T(\mathrm{X})=\bigcup_{n=1}^{\infty} M\left(p_{n, n+1}\right)$, where $M\left(p_{n, n+1}\right)$ denotes the mapping cylinder of $p_{n, n+1}$ : $X_{n+1} \rightarrow X_{n}$. (That is, a topological sum $X_{n} \cup\left(X_{n+1} \times[1 /(n+1), 1 / n]\right), M\left(p_{n, n+1}\right)$ is obtained by identifying points $(x, 1 / n) \in X_{n+1} \times\{1 / n\}$ and $p_{n, n+1}(x) \in X_{n}$ for $x \in X_{n+1}$, and then $T(X)$ is obtained by identifying each point of $X_{n} \times\{1 / n\}$ in $M\left(p_{n-1, n}\right)$ and the corresponding point of $X_{n}$ in $M\left(p_{n, n+1}\right)$.) Put $Z(\mathrm{X})=X \cup T(\mathrm{X})$. Define a function $\mu: Z(\mathrm{X}) \rightarrow I=[0,1]$ by $\mu([x, t])=t$ if $[x, t] \in T(\mathrm{X})$ and $\mu(x)=0$ if $x \in X$. Also, define a retraction $\psi_{t}: Z(X) \rightarrow \mu^{-1}([t, 1])(t \in I)$ by $\psi_{t}(z)=\left[p_{q(t)}(x), t\right]$ for $z=x \in X, \psi_{t}(z)=\left[p_{q(t), n}(x), t\right]$ for $z=[x, s] \in \mu^{-1}((0, t])$ and $x \in X_{n}$, and $\psi_{t}(z)=z$ for $z \in \mu^{-1}([t, 1])$, where $q(t)$ is the natural number such that $1 / q(t) \leqslant t<$ $1 /(q(t)-1)$. The topology of $Z(X)$ is defined by taking as an open base all open sets of $T(X)$ and all the sets of the form $\psi_{(-1 / n)}(U) \cap \mu^{-1}([0,1 / n))$, where $U$ is an open set of $X_{n}(\subset Z(X)), n \geqslant 1$. It may be shown that $Z(X)$ is a compact absolute retract, and that both $\mu$ and $\psi_{t}$ are continuous; for these details, see [4].

Next, we construct a map $f: Z(\mathbf{X}) \rightarrow Z(\mathbf{X})$ such that $f$ is an extension of $g$ : $X \rightarrow X$ and $R(f)=R(g) \cup\{p\}, P(f)=P(g) \cup\{p\}$, where $p=* \in X_{1} \subset Z(\mathrm{X})$. Since $Z(\mathbf{X})$ is an absolute retract, there is an extension $f_{1}: Z(\mathbf{X}) \rightarrow Z(\mathbf{X})$ of $g: X \rightarrow X$. Choose a homeomorphism $h: I \rightarrow I$ such that $h(0)=0, h(1)=1$, and $h(t)>t$ for $0<t<1$, for example, $h(t)=\sqrt{t}$. Define a function $f: Z(\mathrm{X}) \rightarrow Z(\mathrm{X})$ by

$$
f(z)=\psi_{h \cdot \mu(z)}\left(f_{1}(z)\right)
$$

Then $f$ is continuous and $f(p)=p, f \mid X=g$. Also, note that if $z \in Z(\mathbf{X})-(X \cup\{p\})$, then $\mu(z)<\mu(f(z))$, which implies that $R(f)=R(g) \cup\{p\}, P(f)=P(g) \cup\{p\}$. In particular, if $\mathrm{Cl}(R(g)) \neq \mathrm{Cl}(P(g)), \mathrm{Cl}(R(f)) \neq \mathrm{Cl}(P(f))$.

For a special case of the above, we consider the following: Let $X$ be a Cantor set. Take an inverse sequence $X=\left\{X_{n}, p_{n, n+1}\right\}$ such that $X_{1}=\{*\}, X_{n}$ is a finite set for each $n \geqslant 1$, and $X=$ invlim $X$. Then $D=Z(X)$ is a dendrite such that each point $x \in X$ is an end point of $D$. (A point $e$ of a dendrite $X$ is called an end point if there is no subset $A$ of $X$ such that $e \in A$ and $A$ is homeomorphic to the open interval $(0,1)$.) 
An infinite binary tree is a typical dendrite such that the set of end points is a Cantor set (see [3, p.12]). We may assume that $X=C=\{0,1\}^{\infty}$. Then there is a homeomorphism $g: C \rightarrow C$ such that $R(g)=C$ and $P(g)=\phi$. Such a homeomorphism $g$ is constructed as follows: For each $n \geqslant 1$, let $g_{n}:\{0,1\}^{n} \rightarrow\{0,1\}^{n}$ be the cyclic permutation given by $g_{n}\left(a_{1}, \ldots, a_{n}\right)=\left(b_{1}, \ldots, b_{n}\right)$, where $b_{1}=a_{1}+1(\bmod 2)$, for $2 \leqslant i \leqslant n, b_{i}=a_{i}+1$ $(\bmod 2)$ if $a_{i-1}=1$ and $b_{i-1}=0$, and $b_{i}=a_{i}$ otherwise. Let $g: C \rightarrow C$ be given by $g\left(a_{1}, a_{2}, \ldots,\right)=\left(b_{1}, b_{2}, \ldots,\right)$, where $\left(b_{1}, \ldots, b_{n}\right)=g_{n}\left(a_{1}, \ldots, a_{n}\right)$ for all $n \geqslant 1$. Then $g$ is called the binary adding machine. Note that if $x \in C$, then the orbit $\left\{g^{n}(x) \mid n=0,1, \ldots\right\}$ is dense in $C$. In particular, $R(g)=C$ and $P(g)=\phi$. By the above argument, we can obtain a map $f: D \rightarrow D$ such that $R(f)=R(g) \cup\{p\}=$ $X \cup\{p\} \neq\{p\}=P(f)$. In particular, $\mathrm{Cl}(R(f)) \neq \mathrm{Cl}(P(f))$.

\section{REFERENCES}

[1] L. Block and W. A. Coppel, Dynamics in one dimension, Lecture Notes in Math. 1513 (Springer-Verlag, Berlin, Heidelberg, New York, 1992).

[2] E.M. Coven and G.A. Hedlund, ' $\bar{P}=\bar{R}$ for maps of the interval', Proc. Amer. Math. Soc. 79 (1980), 316-318.

[3] G.A. Edgar, Measure, topology, and fractal geometry, UTM (Springer-Verlag, Berlin, Heidelberg, New York, 1990).

[4] J. Krasinkiewicz, 'On a method of constructing ANR-sets. An application of inverse limits', Fund. Math. 92 (1976), 95-112.

[5] S.B. Nadler, Jr., Continuum theory, Pure and applied mathematics 158 (Marcel Dekker, 1992).

[6] H.O. Peitgen and P.H. Richter, The beauty of fractals (Springer-Verlag, Berlin, Heidelberg, New York, Tokyo, 1986).

[7] X.D. Ye, 'The center and the depth of the center of a tree map', Bull. Austral. Math. Soc. 48 (1993), 347-350.

Institute of Matehamtics

University of Tsukubs

Ibarali 305

Japan 